\title{
Brief Review
}

\section{Intensity of reaction to tuberculoprotein is not related to severity of disease in patients with single organ vasculitis of the retina}

\author{
Brijesh Takkar ${ }^{1,2}$, Ramesh C Chauhan ${ }^{3}$, Abhishek Sheemar ${ }^{4}$, Anubha Rathi ${ }^{4}$, Priyanka Sinha ${ }^{1}$, \\ Pradeep Venkatesh ${ }^{4}$ \\ ${ }^{1}$ Department of Ophthalmology, All India Institute of Medical Sciences, Bhopal, India \\ ${ }^{2}$ Smt Kanuri Santhamma Center for Vitreoretinal Diseases, L.V. Prasad Eye Institute, \\ Hyderabad, India \\ ${ }^{3}$ Department of Community and Family Medicine, All India Institute of Medical Sciences, \\ Bhopal, India \\ ${ }^{4}$ Dr R P Centre for Ophthalmic Sciences, All India Institute of Medical Sciences, New Delhi, \\ India
}

Key words: Eales disease, Ocular tuberculosis, Retinal vasculitis, Tuberculoprotein sensitivity, Mantoux test.

\section{Manuscript}

Editor,

Etiology of Eales disease remains elusive and highly disputed despite more than a century of its recognition, with recent suggestions for classifying it as single organ vasculitis of the retina (Sheemar et al, 2018). Pathogenesis has been long believed to involve infectious diseases like Tuberculosis and hypersensitivity to the tuberculoprotein, apart from many other known systemic associations (Biswas et al 2002,2013; Das et al, 2010). However, detailed case-controlled evaluation on this subject has shown no significant association of dermal hypersensitivity to tuberculoprotein (positivity of the Mantoux test) with retinal vasculitis (Biswas et al, 1997, 2013).

Financial Interest: Nil Conflict of Interest: Nil

Received: 01.04.2020

Accepted: 03.06.2020

Corresponding author

Dr. Brijesh Takkar

Consultant, Smt Kanuri Santhamma Center for Vitreoretinal Diseases

L.V. Prasad Eye Institute

Hyderabad, India

E-mail: britak.aiims@gmail.com

Contact: 9868092215
The controversy surrounding role of tuberculoprotein hypersensitivity in Eales disease is in part due to the variability of the Mantoux test reactions, because of reasons including exposure to environmental mycobacteria, immunization for tuberculosis, immune and nutritional status of the patient, and the impact of previous Mantoux tests like boosting, conversion or reversion effects (Chadha, 2001; Menzies, 1999). As it is not possible to control some of these factors in the best of study environments, reading this test as a categorical variable, i.e. positive or negative, may not be the best way to study its impact. The primary objective of this correspondence is to analyze the relation between actual numerical readings of the Mantoux test and severity of Eales disease.

We discuss a post hoc analysis of our previously published study where retinal vasculitis was diagnosed on clinical examination in 200 patients (Sheemar et al, 2018). Past systemic history and duration of disease was recorded during workup. Ultrawide field imaging (UWF) imaging and angiography were done for all the patients (Optos, Dunfermline, UK). Complete 
blood count, erythrocyte sedimentation rate, Mantoux test and chest $x$-ray were done in every case, and further tailored work up was done as needed. Systemic tuberculosis was ruled out in all the patients with Mantoux reading $>14$ $\mathrm{mm}$ with a combination of evidence of acid fast staining for bacilli in sputum, and imaging modalities like computerised tomography of the chest (high resolution and/or contrast enhanced), ultrasound of the abdomen and magnetic resonance imaging of the head. Mantoux test reporting was very carefully screened for quality reporting in each case. To minimize observer bias, reading had been cross checked by doctors in all cases. Cases in which the test had been repeatedly done in the past
6 months were excluded from this analysis to account for the boosting effect.

The clock hours of clinically apparent vasculitis were calculated as centred on the fovea while evaluating the UWF images. More than 3 clock hours of retinal involvement was labelled as severe clinical disease (SCD). The area of capillary non-perfusion (CNP) or retinal ischemia seen on UWF angiography was calculated using the freely available ImageJ software (http://imagej.nih.gov/ij/). Ratio of area of CNP to image area was labelled as proportional retinal ischemia (PRI) (Figure 1). More than $25 \%$ of PRI was categorized as severe retinal ischemia (SRI).

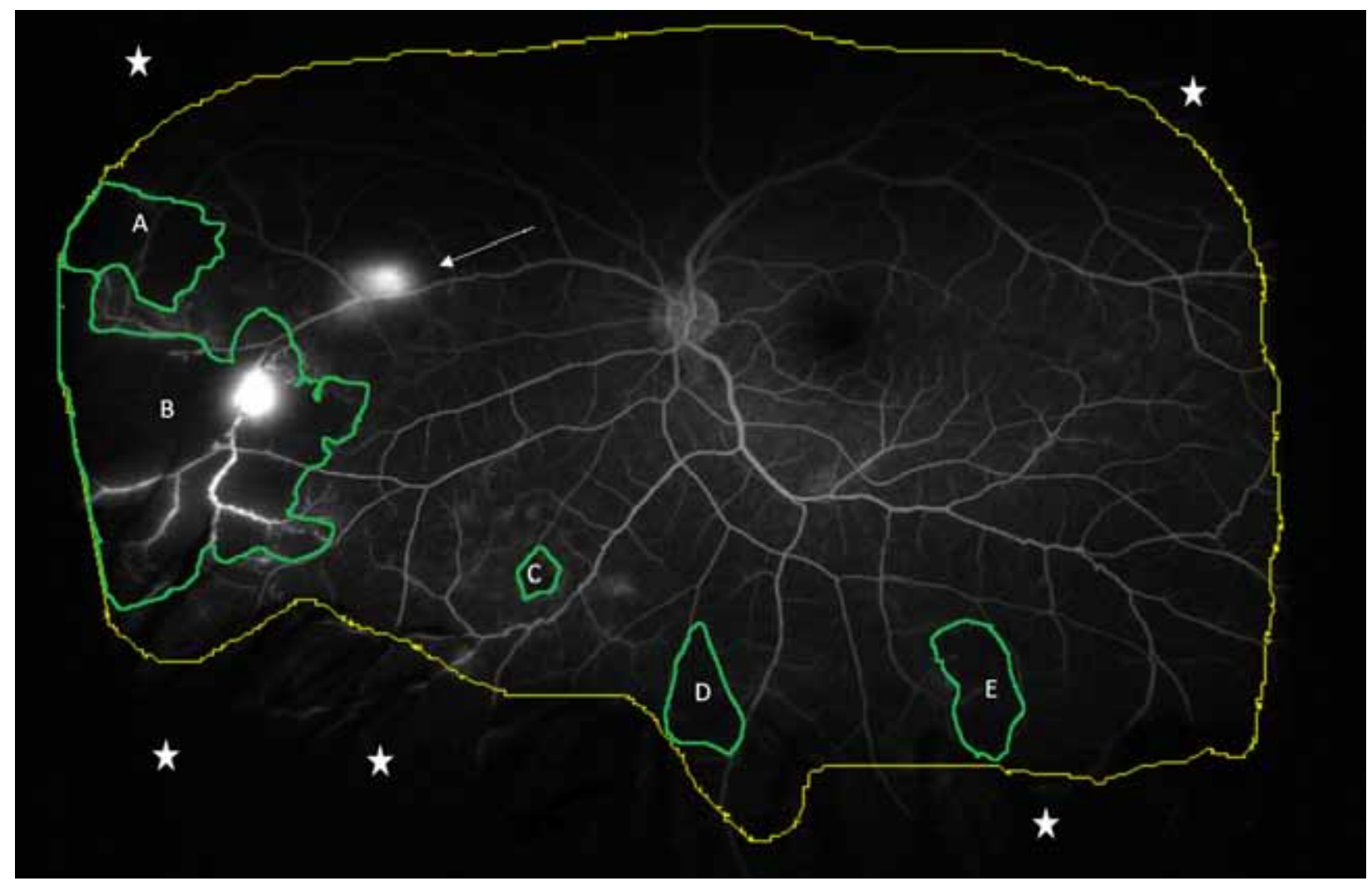

Figure 1: Image shows calculation of proportional retinal ischemia (PRI). Ultrawide field image of a late phase of fluorescein angiogram was obtained and the scale of measurement was set as 1 vertical disc diameter. This was followed by selection of readable areas as demarcated by the yellow line where stars indicate areas removed from calculations due to presence of eye lashes, low brightness or other factors that precluded image assessment. Then the sum of the capillary non-perfusion areas $(\mathrm{A}+\mathrm{B}+\mathrm{C}+\mathrm{D}+\mathrm{E})$, as demarcated by green lines, was obtained and was divided by the total readable image area to calculate the PRI. Two areas of new vessels are also noticeable in this image (arrow). 
Only patients with single organ vasculitis of the retina were included in analysis. Correlation between linear variables was assessed with Pearson's coefficient of correlation, while Spearman's Rho was used for ordinal variables. Groups were created depending on Mantoux reading $(>14$ and $\leq 14)$ and presence or absence of SRI. Chi square test was applied for analysing categorical variables and Independent $t$ test was used to analyse parametric variables. Unadjusted Odds ratio (OR) was assessed using Mantel-Haenszel common OR estimate. Binomial logistic regression was performed to assess the true impact of Mantoux reading on SRI. Only 2 -tailed $\mathrm{p}$ value $<0.05$ was considered to be statistically significant, and all confidence intervals described are true for $95 \%$ of the sample.

Thirty eyes of 20 patients were included for analysis. Details have been summarised in Table 1. SCD was present in $21 / 30(70 \%)$ eyes, while SRI was present in 11/30 (36.7\%) eyes. A weakly negative but statistically insignificant correlation was established between PRI and Mantoux readings $(\mathrm{R}=-0.239, \mathrm{p}=0.203)$. Similarly, Mantoux readings and clock hours of clinical involvement too showed no significant correlation $(\mathrm{R}=-0.099, \mathrm{p}=0.603)$. No significant relationship could be established between Mantoux readings and PRI or SCD (Table 2).

The unadjusted OR estimate of a positive Mantoux with othervariables has been presented in Table 3. None of the tested variables, (SCD, SRI, bilateral disease, new retinal vessels, macular ischemia) showed a high or significant OR. As SRI is indicative of advanced disease, is a precursor of complications and may be associated with poor outcomes, a multivariate analysis was performed to judge the true impact of Mantoux readings on it. The adjusted OR of a positive Mantoux reading being associated with SRI was found to be $0.472(\mathrm{p}=0.43$, $\mathrm{CI}=0.07-3.08$ ).
Previously, tissue samples retrieved from patients of retinal vasculitis have been shown to contain DNA of mycobacterium tuberculosis (Biswas et al, 1999, Madhvan et al, 2000), but the organism has not been grown on culture. Polymerase chain reaction (PCR) positivity of ocular fluids for Mycobacterium has also been used as a surrogate marker of infection by some author groups (Gupta et al, 2001; Singh et al 2012). Considering the possibility of Eales disease being a hypersensitivity response rather than a true infectious vasculitis, one would expect some degree of correlation between the dermal and retinal hypersensitivity. However, we could not identify any significant correlation or association between Mantoux readings and retinal vasculitis- whether by evaluating the clinical extent of vasculitis or by charting ischemic area on UWF. Further, the ORs of test positivity for severe disease reached borderline significance and were very low, with lower confidence levels much below 1 and very large confidence bounds.

Our analysis is limited mainly by its sample size and the use of both eyes of 10 patients in the analysis. Though our stringent exclusion criteria decreased the sample size to nearly $10 \%$ of the 200 screened cases making it underpowered, it was necessary to maintain uniformity. A previous study on PCR positive tubercular retinal vasculitis had shown that Mantoux may be strongly positive even when PCR of ocular fluids was negative (Gupta et al, 2001). Another study from Eastern India, had found Mantoux to be positive in $30 \%$ of their sample, but the authors could prove tuberculosis only in $5 \%$ of the cases (Saurabh et al, 2011). Keeping in mind these and our results, we recommend evaluating the role of tuberculin test in retinal vasculitis with larger sized studies, as it is likely to have implications on actual benefits of presumptive treatment with anti-Tubercular drugs in these patients. 
Table 1: Distribution of variables

\begin{tabular}{|l|l|}
\hline Number of eyes & $\mathbf{3 0}$ \\
\hline Number of patients & 20 \\
\hline Mean age & $22.03+/-10.58$ yrs \\
\hline Male : Female ratio & $7: 3$ \\
\hline Duration of disease & $5.2+/-7.2$ months \\
\hline Mean LogMAR visual acuity & $0.68+/-0.92$ LogMAR units \\
\hline Venous: Mixed vessel disease & $9: 1$ \\
\hline Mean clock hours of clinical involvement & $5.83+/-3.42$ \\
\hline Mean of clock hours of angiographic ischemia & $3.9+/-2.91$ \\
\hline Mean Mantoux reading & $16.03+/-6.71$ mm (range- $7-31)$. \\
\hline Presence of new vessels & $17 / 30(56 \%)$ \\
\hline Presence of macular ischemia & $4 / 30(13.3 \%)$ \\
\hline Mean Proportion of retinal ischemia & $0.23+/-0.21$ \\
\hline
\end{tabular}

Table 2: Relation between Mantoux findings and retinal vasculitis

\begin{tabular}{|l|l|l|}
\hline Category (n) & Mean mantoux reading (mm) & P value \\
\hline Proportional retinal ischemia & $17.37+/-7.07$ & \\
Non severe (19) & $13.73+/-5.6$ & 0.15 \\
Severe (11) & & \\
\hline Clock hours of clinical disease & $14.78+/-6.82$ & 0.51 \\
Non severe (9) & $16.57+/-6.76$ & \\
Severe (21) & Mantoux reading $>15(\mathrm{n})$ & \\
\hline & $12(63.2 \%)$ & 0.05 \\
\hline Proportional retinal ischemia & $3(27.3 \%)$ & \\
Non severe (19) & $4(44.4 \%)$ & 0.69 \\
Severe (11) & $11(52.4 \%)$ & \\
\hline Clock hours of clinical disease & & \\
Non severe (9) & & \\
Severe (21) &
\end{tabular}

Table 3: Odds ratio estimates for association with positive Mantoux reading

\begin{tabular}{|l|l|l|l|}
\hline Variable & Odds ratio & Confidence interval* & P value \\
\hline Severe clinical disease & 1.37 & $0.29-6.6$ & 0.69 \\
\hline $\begin{array}{l}\text { Severe retinal ischemia } \\
\text { (unadjusted OR) }\end{array}$ & 0.22 & $0.04-1.1$ & 0.05 \\
\hline Bilateral disease & 0.29 & $0.05-1.45$ & 0.121 \\
\hline Retinal new vessels & 0.24 & $0.05-1.13$ & 0.06 \\
\hline Macular ischemia & 0.28 & $0.03-3.13$ & 0.05 \\
\hline $\begin{array}{l}\text { Severe retinal ischemia } \\
\text { (adjusted OR)** }\end{array}$ & 0.47 & $0.07-3.08$ & 0.43 \\
\hline $\begin{array}{l}\text { OR: Odds ratio. * Confidence intervals are true for 95\% of values. } \\
* * \text { Adjustment done with regression analysis for duration, type of retinal vessel involved, } \\
\text { anterior or posterior disease and severe clinical disease. }\end{array}$ \\
\hline
\end{tabular}




\section{References}

Biswas J, Narain S, Roy S, Madhavan HN (1997). Evaluation of lymphocyte proliferation assay to purified protein derivative, enzyme linked immunosorbent assay, and tuberculin hypersensitivity in Eales disease. Indian $\mathrm{J}$ Ophthalmol. 45:93-7.

Biswas J, Therese L, Madhavan HN (1999). Use of polymerase chain reaction in detection of mycobacterium tuberculosis complex DNA from vitreous sample of Eales disease. Br J Ophthalmol. 83:994-7.

Biswas J, Sharma T, Gopal L, Madhavan HN, Sulochana KN, Ramakrishnan S (2002). Eales disease - an update. Surv ophthalmol. 47(3):197-214.

Biswas J, Ravi RK, Naryanasamy A, Kulandai LT, Madhavan HN (2013). Eales' disease-current concepts in diagnosis and management. $\mathrm{J}$ ophthalmic inflamm infect. 3(1):11.

Chadha VK. Tuberculin test (2001). Indian J Pediatr. 2001;68(1):53-8.

Das T, Pathengay A, Hussain N, Biswas $J$ (2010). Eales' disease: diagnosis and management. Eye. 24(3):472.
Gupta A, Gupta V, Arora S, Dogra MR, Bambery P (2001). PCR-positive tubercular retinal vasculitis: Clinical characteristics and management. Retina. 21(5):435-44.

Madhavan HN, Therese KL, Gunisha $\mathrm{P}$, et al (2000). Polymerase chain reaction for detection of Mycobacterium tuberculosis in epiretinal membrane in Eales disease. Invest Ophthalmol Vis Sci 41:822-5.

Menzies D. Interpretation of repeated tuberculin tests: boosting, conversion, and reversion (1999). Am J respircrit care med. 159(1):15-21.

Saurabh K, Das RR, Biswas J, Kumar A (2011). Profile of retinal vasculitis in a tertiary eye care center in Eastern India. Ind J Ophthalmol. 59(4):297.

Sheemar A, Takkar B, Temkar S, Sood R, Chawla R, Venkatesh P (2018). Retinal Vasculitis Is Principally a Single-Organ Vasculitis of the Eye. J Clin Rheumat. 24(2):879.

Singh R, Toor P, Parchand S, Sharma K, Gupta V, Gupta A (2012). Quantitative polymerase chain reaction for Mycobacterium tuberculosis in so-called Eales' disease. Ocul Immunol Inflamm. 20(3):153-7. 